\title{
Pion form factor from local-duality QCD sum rule
}

\section{Wolfgang Lucha}

Institute for High Energy Physics, Austrian Academy of Sciences, Nikolsdorfergasse 18, A-1050,

Vienna, Austria

E-mail: Wolfgang.Lucha@oeaw.ac.at

\section{Dmitri Melikhov*}

Institute for High Energy Physics, Austrian Academy of Sciences, Nikolsdorfergasse 18, A-1050,

Vienna, Austria, and

D. V. Skobeltsyn Institute of Nuclear Physics, Moscow State University, 119991, Moscow, Russia

E-mail: dmitri_melikhovegmx.de

We present our recent results for the pion elastic form factor [1] obtained within a local-duality three-point sum rule (a Borel sum rule in the limit of an infinite Borel parameter). Our analysis [1] includes the $O(1)$ and $O\left(\alpha_{s}\right)$ contributions and is therefore applicable in a broad range of spacelike momentum transfers. Our results demonstrate in essentially model-independent way that the $O(1)$ term, which provides the subleading $1 / Q^{4}$ power correction at asymptotically large momentum transfers, contributes more than half of the pion form factor in the region $Q^{2} \leq 20$ $\mathrm{GeV}^{2}$. To probe the accuracy of local-duality sum rules for form factors, we apply precisely the same procedures to extract the form factor in a quantum-mechanical potential model. Comparison of the exact form factor known in this model with the result of the sum-rule calculation gives a probe of the systematic error of the method. In our example this error is found to be at the level of $10-20 \%$. We expect similar systematic errors for form factors obtained from local-duality sum rules in QCD.

8th Conference Quark Confinement and the Hadron Spectrum

September 1-6, 2008

Mainz, Germany

\footnotetext{
${ }^{*}$ Speaker.
} 


\section{Introduction}

The values of hadron parameters (in particular, form factors) extracted from QCD sum rules depend on two ingredients: (i) the field-theoretic calculation of the relevant correlator and (ii) the technical "extraction procedure", which is external to the underlying field theory. The second ingredient introduces a systematic error which is very hard to control in any version of QCD sum rules [2]. So, in order to probe the accuracy of the sum-rule predictions it is plausible to apply different versions of sum rules to the same quantities and compare the results.

In this talk we report our recent analysis of the pion form factor [1] within the so-called localduality (LD) version of three-point QCD sum rules (for details of this method see [3] and references therein) and try to get an idea of the expected accuracy of this calculation.

The LD sum rules are the Borel sum rules in the limit of an infinitely large Borel parameter. For the relevant choice of the pion interpolating current, the condensate contributions to the correlators vanish in this limit and the pion observables are given by dispersion integrals via the spectral densities of purely perturbative QCD diagrams. The integration region in the dispersion integrals is restricted to the pion "duality interval".

This approach has the following attractive features: (i) it is applicable in a broad range of momentum transfers, and (ii) it does not refer directly to the pion distribution amplitude. Therefore, it allows us to study in a relatively model-independent way the interplay between perturbative and nonperturbative dynamics in the pion from factor at intermediate momentum transfers.

\section{Sum rule}

Let us consider the sum rules for the pion form factor and the decay constant in the LD limit, where all condensate contributions vanish. According to the standard assumption that the groundstate contribution is dual to the low-energy region of the free-quark diagrams, for the case of massless quarks we obtain to $\alpha_{s}$ accuracy

$$
\begin{aligned}
F_{\pi}^{\mathrm{LD}}\left(Q^{2}\right)\left(f_{\pi}^{\mathrm{LD}}\right)^{2} & =\frac{1}{\pi^{2}} \int_{0}^{s_{0}} d s_{1} \int_{0}^{s_{0}} d s_{2}\left[\Delta^{(0)}\left(s_{1}, s_{2}, Q^{2}\right)+\alpha_{s} \Delta^{(1)}\left(s_{1}, s_{2}, Q^{2}\right)+O\left(\alpha_{s}^{2}\right)\right], \\
\left(f_{\pi}^{\mathrm{LD}}\right)^{2} & =\frac{1}{\pi} \int_{0}^{s_{0}} d s\left[\rho^{(0)}(s)+\alpha_{s} \rho^{(1)}(s)+O\left(\alpha_{s}^{2}\right)\right] .
\end{aligned}
$$

The double dispersion representation (2.1) for $F_{\pi} f_{\pi}^{2}$, even in the LD limit, has two essential ambiguities: (a) The choice of the shape of the duality region in the $s_{1}-s_{2}$ plane: the simplest choice, implemented in (2.1), is a square, but any other region symmetric under $s_{1} \leftrightarrow s_{2}$ may be chosen. (b) The duality interval $s_{0}$ in the 3-point correlator may (and should) depend on $Q^{2}$. This dependence has been neglected. Now, we remind the reader that sum rules are predictive only if one imposes a criterion to fix the effective continuum threshold [2]. In (2.1) we have made an additional essential assumption - we have set the parameter $s_{0}$ to be the same for both sum rules (2.1) and (2.2). The form factor (2.1) has the following attractive properties:

(i) It satisfies the normalization condition $F_{\pi}\left(Q^{2}=0\right)=1$ due to the vector Ward identity, which provides the relation between the spectral density of the self-energy diagram and the double spectral 
density of the triangle diagram at zero momentum transfer:

$$
\lim _{Q^{2} \rightarrow 0} \Delta^{(i)}\left(s_{1}, s_{2}, Q^{2}\right)=\pi \rho^{(i)}\left(s_{1}\right) \delta\left(s_{1}-s_{2}\right), \quad \rho^{(0)}(s)=\frac{1}{4 \pi}, \quad \rho^{(1)}(s)=\frac{1}{4 \pi^{2}} .
$$

Clearly, for consistency one should then take into account the radiative corrections to the same order in the sum rules for two- and three-point correlators.

(ii) For $Q^{2} \gg s_{1}, s_{2}$, one finds $\Delta^{(0)}\left(s_{1}, s_{2}, Q^{2}\right) \rightarrow 3\left(s_{1}+s_{2}\right) / 2 Q^{4}, \Delta^{(1)}\left(s_{1}, s_{2}, Q^{2}\right) \rightarrow 1 / 2 \pi Q^{2}$ [1]. Substituting these relations into (2.1) and identifying $f_{\pi}^{\mathrm{LD}}$ with the pion decay constant $f_{\pi}$ yields at large $Q^{2}$ :

$$
F_{\pi}^{\mathrm{LD}}\left(Q^{2}\right)=\frac{8 \pi f_{\pi}^{2} \alpha_{s}}{Q^{2}}+\frac{96 \pi^{4} f_{\pi}^{4}}{Q^{4}}+O\left(\alpha_{s} f_{\pi}^{4} / Q^{4}\right)+O\left(\alpha_{s}^{2}\right) .
$$

Interestingly, we have obtained the correct asymptotic behaviour of the pion form factor in the limit $Q^{2} \rightarrow \infty$ (up to the running of $\alpha_{s}$ ). This property is due to (i) the factorization of the $O\left(\alpha_{s}\right)$ cut triangle diagram at large $Q^{2}$ and (ii) choosing the same value of $s_{0}$ in 2- and 3-point correlators.

However, there are also obvious problems:

First, the sum rule cannot be directly applied at small $Q^{2}$, although the expression (2.1) leads to the correct normalization of the form factor: Recall that the OPE for the three-point correlator was obtained in the region where all three external variables $\left|p_{1}^{2}\right|,\left|p_{2}^{2}\right|$, and $Q^{2}$ are large. [A technical indication of the fact that the LD sum rule (2.1) cannot be applied at very small $Q^{2}$ is the presence of terms $\sim \sqrt{Q^{2}}$ leading to an infinite value of the pion radius.] Moreover, the spectral density $\Delta\left(s_{1}, s_{2}, Q^{2}\right)$ contains the terms $O(1)$ and $O\left(\alpha_{s}\right)$, whereas higher powers are unknown. Since the coupling constant $\alpha_{s}$ is not small in the soft region, our spectral density is not sufficient for application to the form factor at $Q^{2} \leq 1 \mathrm{GeV}^{2}$.

Second, in order to apply the obtained formulas for large $Q^{2}$, higher-order radiative corrections, leading to the running of $\alpha_{s}$, should be taken into account. Such an accuracy is beyond our two-loop calculation; nevertheless, a self-consistent expression for the form factor applicable for all $Q^{2}>0$ may be obtained from (2.1) by using in the sum rule for $F_{\pi}$ the $Q^{2}$-dependent threshold

$$
s_{0}\left(Q^{2}\right)=\frac{4 \pi^{2} f_{\pi}^{2}}{1+\alpha_{s}\left(Q^{2}\right) / \pi} .
$$

One should understand the meaning of $\alpha_{s}\left(Q^{2}\right)$ at small $Q^{2}$ : in [3] it was argued that $s_{0}$ is the relevant scale of $\alpha_{s}$ in the LD sum rules for the decay constant and for the form factor at $Q^{2}=0$. For the calculations we have assumed the freezing of $\alpha_{s}$ at the value 0.3 .

The results for the pion form factor are shown in Fig. 1 a, and Fig. 1b presents the ratio of the $O(1)$ and the $O\left(\alpha_{s}\right)$ contributions to the form factor vs $Q^{2}$ for different models of the effective continuum thresholds. One can clearly see that the ratio is mainly determined by the corresponding double spectral densities $\Delta^{(0)}$ and $\Delta^{(1)}$, whereas its sensitivity to the effective continuum threshold is rather weak.

Obviously, the $O(1)$ term, which provides the subleading $1 / Q^{4}$ power correction at large $Q^{2}$, dominates the form factor at low $Q^{2}$, and still gives $50 \%$ at $Q^{2}=20 \mathrm{GeV}^{2}$. The $O\left(\alpha_{s}\right)$ term gives more than $80 \%$ of the form factor only above $Q^{2}=100 \mathrm{GeV}^{2}$. Such a pattern of the pion form factor behaviour has been conjectured many times in the literature; we now obtain this behaviour in an explicit calculation. This analysis supports the results of [4] obtained by inclusion of $O(1)$ and $O\left(\alpha_{s}\right)$ terms within the dispersion approach. 


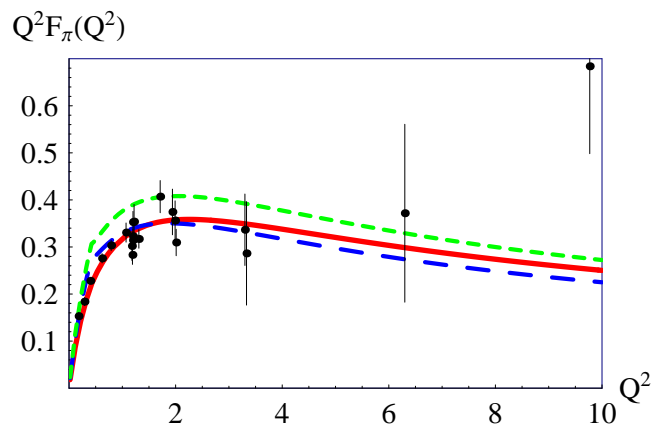

(a)

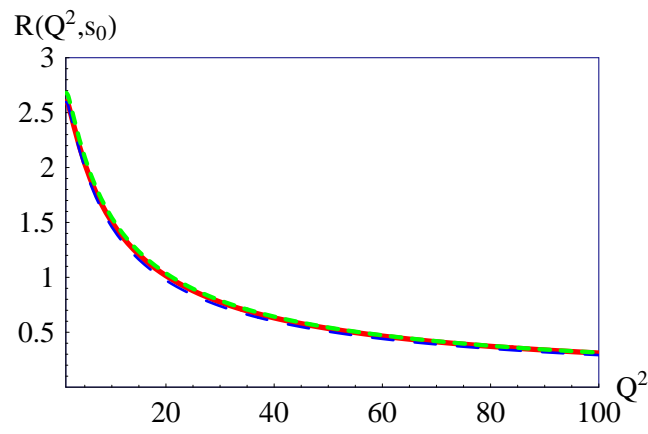

(b)

Figure 1: (a) The pion form factor vs $Q^{2}$ (in units of $\mathrm{GeV}^{2}$ ) for $Q^{2} \geq 0.5 \mathrm{GeV}^{2}$. Experimental data from [5]. (b) The ratio of the $O(1) / O\left(\alpha_{s}\right)$ contributions to the form factor. Solid (red) line: the result of the calculation according to (2.1); short-dashed (green) line: the form factor obtained with constant $s_{0}=0.65$ $\mathrm{GeV}^{2}$; long-dashed (blue) line: $s_{0}=0.6 \mathrm{GeV}^{2}$.

\section{Discussion and Conclusions}

We presented the results of the first analysis of the pion form factor which takes into account both the $O(1)$ and the $O\left(\alpha_{s}\right)$ contributions within the LD sum rule [1]. These ingredients are crucial for the possibility to consider the form factor in a broad range of $Q^{2}$ and to study the transition from the nonperturbative to the perturbative region.

Let us summarize the essential ingredients and the lessons to be learnt from our analysis:

1. We have included the exact $O(1)$ and $O\left(\alpha_{s}\right)$ terms into the spectral representation for the form factor, and omitted the $O\left(\alpha_{s}^{2}\right)$ terms, which are expected to contribute at the level of less than $\sim 10 \%$.

2. The numerical results for the form factor from the LD sum rule depend sizeably on the model for the effective continuum threshold used for the calculations: this very quantity determines to a

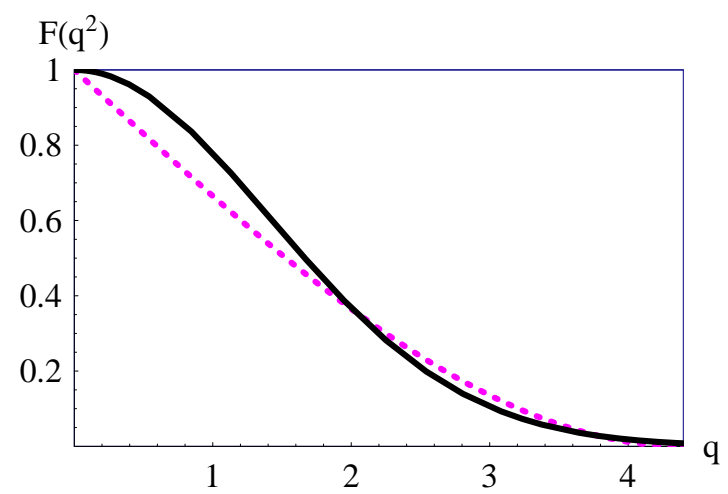

Figure 2: The form factor obtained from the LD sum rule in a harmonic-oscillator quantum-mechanical model. Solid line: exact form factor, dotted line: the form factor obtained from the LD sum rule with a constant effective continuum threshold fixed from the known value of the decay constant of the ground state. 
great extent the value of the extracted form factor. The possibility to fix this effective continuum threshold is the weak point of the approaches based on all versions of sum rules, which limits their predictivity [2]. We used the same threshold in the two- and three-point sum rules considered; this allows us to relate the value of the threshold to the pion decay constant, known experimentally. In this way we have no free numerical parameters in our analysis. However, our analysis is obviously not free from systematic uncertainties.

3. One cannot assign any rigorous error to the obtained form factor. In order to get an idea of the corresponding accuracy, we have used precisely the same algorithms within the non-relativistic harmonic-oscillator model, where the exact form factor may be calculated independently, and found the error to be at a 10-20\% level (see Fig. 2). We believe a similar estimate to be true for our QCD calculation.

4. We can control rather well the relative weights of the $O(1)$ and $O\left(\alpha_{s}\right)$ contributions to the form factor: their ratio is practically independent of the continuum threshold. Thus, our results convincingly show that the $O\left(\alpha_{s}\right)$ contribution to the pion form factor remains below $50 \%$ at $Q^{2} \leq$ $20 \mathrm{GeV}^{2}$; these results definitely speak against the pQCD approach to exclusive processes, which assumes the form factor to be described by the $O\left(\alpha_{s}\right)$ term already at $Q^{2}$ of the order of several $\mathrm{GeV}^{2}$.

\section{Acknowledgments}

We are grateful to Victor Braguta for collaboration on this subject and to Alexander Bakulev and Silvano Simula for valuable discussions. D. M. was supported by the Austrian Science Fund (FWF) under project P20573 and by RFBR under project 07-02-00551.

\section{References}

[1] V. Braguta, W. Lucha, and D. Melikhov, Pion form factor at spacelike momentum transfers from local-duality QCD sum rule, Phys. Lett. B 661 (2008) 354.

[2] W. Lucha, D. Melikhov, and S. Simula, Systematic uncertainties of hadron parameters obtained with QCD sum rules, Phys. Rev. D 76 (2007) 036002; Can one control systematic errors of QCD sum-rule predictions for bound states? Phys. Lett. B 657 (2007) 148; Study of systematic errors of bound-state parameters in SVZ sum rules, Phys. Atom. Nucl. 71 (2008) 1461; Accuracy of bound-state form factors extracted from dispersive sum rules, arXiv:0810.1920; D. Melikhov, Hadron form factors from sum rules for vacuum-to-hadron correlators, arXiv:0810 . 4497; W. Lucha and D. Melikhov, Quark-hadron duality and hadron properties from correlators of pseudoscalar and axial currents, Phys. Rev. D 73 (2006) 054009; OPE and sum rules for correlators of pseudoscalar and axial currents, Phys. Atom. Nucl. 70 (2007) 891.

[3] A. V. Radyushkin, Quark-hadron duality and intrinsic transverse momentum, Acta Phys. Polon. B 26 (1995) 2067.

[4] V. V. Anisovich, D. I. Melikhov, and V. A. Nikonov, Quark structure of the pion and pion form factor, Phys. Rev. D 52 (1995) 5295; Photon-meson transition form factors at low and moderately high $Q^{2}$, Phys. Rev. D 55 (1997) 2918; D. Melikhov, Dispersion approach to quark binding effects in weak decays of heavy mesons, Eur. Phys. J. direct C4 (2002) 2 [hep-ph/ 0110087 ].

[5] C. J. Bebek, Electroproduction of single pions at low epsilon and a measurement of the pion form-factor up to $Q^{2}=10 \mathrm{GeV}^{2}$, Phys. Rev. D 17 (1978) 1693. 